\title{
Strengthening the open cluster distance scale via VVV photometry ${ }^{\star}$
}

\author{
D. Majaess ${ }^{1}$, D. Turner ${ }^{1}$, C. Moni Bidin ${ }^{2}$, D. Geisler ${ }^{2}$, J. Borissova ${ }^{7}$, D. Minniti ${ }^{3,4,5}$, C. Bonatto ${ }^{9}$, W. Gieren ${ }^{2}$, \\ G. Carraro ${ }^{8}$, R. Kurtev ${ }^{7}$, F. Mauro ${ }^{2}$, A.-N. Chené ${ }^{2,7}$, D. Forbes ${ }^{10}$, P. Lucas ${ }^{6}$, I. Dékány ${ }^{2}$, R. K. Saito ${ }^{3}$, and M. Soto ${ }^{11}$ \\ ${ }^{1}$ Department of Astronomy and Physics, Saint Mary's University, Halifax, NS B3H 3C3, Canada \\ e-mail: dmajaess@cygnus.smu.ca \\ 2 Departmento de Astronomía, Universidad de Concepción, Casilla 160-C, Concepción, Chile \\ 3 Departamento Astronomía y Astrofísica, Pontificia Universidad Católica de Chile, Av. Vicuña Mackenna 4860, Santiago, Chile \\ 4 Vatican Observatory, 00120 Vatican City State, Italy \\ 5 Department of Astrophysical Sciences, Princeton University, Princeton, NJ 08544-1001, USA \\ ${ }^{6}$ Centre for Astrophysics Research, University of Hertfordshire, College Lane, Hatfield AL10 9AB, UK \\ 7 Departamento de Física y Astronomía, Facultad de Ciencias, Universidad de Valparaíso, Ave. Gran Bretaña 1111, Playa Ancha, \\ Casilla 5030, Valparaíso, Chile \\ ${ }^{8}$ European Southern Observatory, Ave. Alonso de Cordova 3107, Casilla 19, 19001 Santiago, Chile \\ 9 Departamento de Astronomia, Universidade Federal do Rio Grande do Sul, Av. Bento Gonalves 9500 Porto Alegre 91501-970, \\ RS, Brazil \\ ${ }^{10}$ Department of Physics, Sir Wilfred Grenfell College, Memorial University, Corner Brook, Newfoundland A2H 6P9, Canada \\ 11 Departamento de Física, Universidad de La Serena, Cisternas 1200 Norte, La Serena, Chile \\ Received 9 December 2011 / Accepted 18 December 2011
}

\section{ABSTRACT}

\begin{abstract}
Approximately $14 \%$ of known Galactic open clusters possess absolute errors $\leq 20 \%$ as evaluated from $n \geq 3$ independent distance estimates, and the statistics for age estimates are markedly worse. That impedes such diverse efforts as calibrating standard candles and constraining masses for substellar companions. New data from the VVV survey may be employed to establish precise cluster distances with comparatively reduced uncertainties $(\leq 10 \%)$. This is illustrated by deriving parameters for Pismis 19 and NGC 4349 , two pertinent open clusters which hitherto feature sizable uncertainties $(60 \%)$. Fundamental parameters determined for Pismis 19 from new VVV $J H K_{\mathrm{s}}$ photometry are $d=2.40 \pm 0.15 \mathrm{kpc},\left\langle E_{J-H}\right\rangle=0.34 \pm 0.04$, and $\log \tau=9.05 \pm 0.10$, whereas for NGC 4349 the analysis yielded $d=1.63 \pm 0.13 \mathrm{kpc}, E_{J-H}=0.09 \pm 0.02, \log \tau=8.55 \pm 0.10$. The results exhibit a significant $(\geq 5 \times)$ reduction in uncertainties, and indicate that: i) existing parameters for the substellar object NGC 4349 127b require revision, in part because the new cluster parameters imply that the host is $20 \%$ less-massive $\left(\mathcal{M}_{*} / \mathcal{M}_{\odot} \sim 3.1\right)$; ii) R Cru is not a member of NGC 4349 and should be excluded from period-Wesenheit calibrations that anchor the distance scale; iii) and results for Pismis 19 underscore the advantages gleaned from employing deep VVV $J H K_{\mathrm{s}}$ data to examine obscured $\left(A_{V} \sim 4\right)$ and differentially reddened intermediate-age clusters.
\end{abstract}

Key words. techniques: photometric - Hertzsprung-Russell and C-M diagrams - dust, extinction - stars: distances

\section{Introduction}

Approximately $30 \%$ of the 395 open clusters featuring $n \geq 3$ independent distance estimates exhibit absolute errors $\geq 20 \%$ (Paunzen \& Netopil 2006, their Fig. 2). There are $\geq 2 \times 10^{3}$ cataloged Galactic open clusters (Dias et al. 2002), implying that merely $\sim 14 \%$ of the known sample possess errors $\leq 20 \%$ as evaluated from three distance estimates. The uncertainties permeate into analyses which rely on the cluster zero-point, such as the calibration of any constituent standard candles or substellar companions (Lovis \& Mayor 2007; Majaess et al. 2011b). Consider that published parameters for NGC 4349 span $d=900-2200$ pc and $\tau=0.1-0.6$ Gyr (Sect. 3.2). Yet physical parameters for the substellar companion to TYC 8975-2601-1 (Lovis \& Mayor 2007; Kashyap et al. 2008) rely on those inferred for the host from cluster membership (NGC 4349). Furthermore, the nearer distance and younger age for NGC 4349 potentially imply cluster membership for the classical Cepheid R Cru, which lies within the cluster's corona. Establishing cluster member-

* Based on observations taken within the ESO VISTA Public Survey VVV, Programme ID 179.B-2002. ship would enable the subsequent calibration of Cepheid periodluminosity and period-Wesenheit relations (Turner 2010). Such functions bolster efforts to establish extragalactic distances and zero-point the SNe Ia scale (e.g., Pietrzyński \& Gieren 2004). The aforementioned examples underscore the broad ramifications of an uncertain cluster scale. Admittedly, age estimates for open clusters are less reliable since a third exhibit absolute errors $>50 \%$ (Paunzen \& Netopil 2006, $n \geq 3$ ), and presumably the statistics worsen for obscured clusters near the Galatic plane.

In this study, new VVV (VISTA Variables in the Vía Láctea) $J H K_{\text {s }}$ photometry is employed to illustrate the marked improvement that can be achieved vis à vis open cluster distances. Two important clusters featuring particularly discrepant published parameters are examined, namely Pismis 19 and NGC 4349. Distances for the clusters display a $~ 60 \%$ spread and individual uncertainties of $\sim 30 \%$. Efforts to secure precise parameters for Pismis 19 via optical photometry have been complicated by differential reddening and $A_{V} \sim 4$ mag of obscuring dust. Parameters for Pismis 19 and NGC 4349 derived here exhibit a marked $(>5 \times)$ reduction in uncertainties (Sect. 3 ), and highlight 


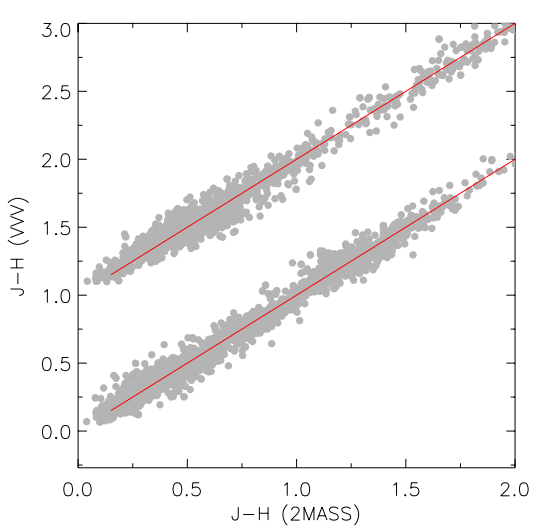

Fig. 1. A comparison between $2 \mathrm{MASS}$ and VVV $J-H$ photometry for the regions encompassing Pismis 19 and NGC 4349. A 1:1 correlation (red line) exists to within the uncertainties. The data for Pismis 19 were deliberately offset from zero for presentation purposes. Pertinent details regarding the pipeline employed here to process the VVV data are described in Moni Bidin et al. (2011) and Mauro et al. (in prep.).

the advantages of using VVV data to determine reliable cluster distances and compliment existing efforts.

\section{VVV photometry}

The VVV survey aims to establish precise multi-epoch $J H K_{\mathrm{s}}$ photometry for fields in the Galactic bulge and near the Galactic plane $\left(\ell=295-10^{\circ}\right.$, Minniti et al. 2010; Catelan et al. 2011; Saito et al. 2011). VVV images exhibit increased angular resolution relative to $2 \mathrm{MASS}$, and extend $\sim 4$ mag fainter for Galactic disk stars. The deep $J H K_{\mathrm{s}}$ photometry facilitates isochrone fitting by revealing the target cluster's evolutionary morphology, which is particularly important when investigating highly reddened clusters. The VVV survey will provide standardized (2MASS) $J H K_{\mathrm{s}}$ photometry for stars in $\geq 3 \times 10^{2}$ open clusters and $\geq 39$ globular clusters (e.g., M28). Details of the pipeline constructed to process and extract the VVV photometry employed here are discussed in Mauro et al. (in prep.). PSF photometry was performed using DAOPHOT and subsequently tied to 2MASS $J H K_{\mathrm{s}}$ standards (Fig. 1, see also Moni Bidin et al. 2011). However, as with any nascent large scale survey adjustments to the zero-point may occur as improvements and systematic errors are identified.

\subsection{The advantages of $\mathrm{JHK}_{\mathrm{s}}$ photometry}

Precise $J H K_{\mathrm{S}}$ observations of stellar clusters are desirable since total and differential reddening are less deleterious than in the optical $\left(A_{J} \sim 0.2 \times A_{V}\right)$. Sizable extinction may shift a significant fraction of the main-sequence near/beyond the limiting magnitude where uncertainties are largest. Consider that merely $\sim 3^{\mathrm{m}}$ of the cluster sequence for Pismis 19 was sampled in existing optical surveys owing to significant reddening, which subsequently complicated efforts to establish the cluster zero-point. The $J H K_{\mathrm{S}}$ reddening vector provides viable solutions for the intrinsic colors of stars across much of the main-sequence. The $J H K_{\mathrm{s}}$ reddening vector can be determined from red clump stars along the line of sight (Straižys \& Laugalys 2008; Majaess et al. 2011b), and the ratio of total-to-selective extinction can be inferred in certain instances using red clump stars via the variable extinction method (e.g., Majaess et al. 2011b). Straižys \& Laugalys (2008) demonstrated that $E(J-H) / E\left(H-K_{\mathrm{s}}\right)$ is (rather) constant for dust occupying the inner Galaxy. A consensus exists that any variations in the infrared would be marginal relative to that expected for the optical.
$J H K_{\mathrm{s}}$ photometry is particularly suited for detecting and characterizing the heavily obscured pre main-sequence population of young clusters (Bonatto \& Bica 2010). For later-type stars, $J H K_{\mathrm{s}}$ photometry is relatively insensitive to variations in chemical composition (e.g., the Hyades anomaly, Turner 1979; Majaess et al. 2011a; see also Straižys \& Lazauskaitė 2009). That claim is supported in part by the establishment of seven benchmark open clusters $(d<250$ pc) which exhibit matching $J H K_{\mathrm{s}}$ ZAMS and revised Hipparcos distances (the Hyades, $\alpha$ Per, Praesepe, Coma Ber, IC 2391, IC 2609, and NGC 2451, van Leeuwen 2009; Majaess et al. 2011a). The zero-point of the Padova isochrone employed (Sect. 3) matches that scale, to within the uncertainties. Isochrones, models, and the distance scale should be anchored (\& evaluated) using clusters where consensus exists, rather than the discrepant case (i.e. the Pleiades). The 2MASS survey provides invaluable all-sky $J H K_{\mathrm{s}}$ photometric standards. A similar survey tied to JohnsonCousins $U B V R I$ photometry is desirable. $U$-band photometry is particularly challenging to standardize and zero-point errors are common (Sect. 3.2, see also Cousins \& Caldwell 2001). However, $U B V$ color-color analyses permit crucial dereddening for younger stars.

In summary, the VVV survey is aptly tailored to foster cluster research (Minniti et al. 2011; Borissova et al. 2011; Moni Bidin et al. 2011; Majaess et al. 2011b). Admittedly, acquiring precise and standardized $U B V J H K_{\mathrm{s}}$ photometry is ideal, and enables the characterization of potential systemic errors. $U B V$ data by Turner/Forbes (unpublished) and Carraro (2011, in press) are employed to corroborate parameters determined via the VVV photometry.

\section{Analysis}

\subsection{Pismis 19}

Pismis 19 is a heavily reddened open cluster (Piatti et al. 1998; Carraro \& Munari 2004). The cluster's non-symmetric appearance in optical images is indicative of differential reddening. Piatti et al. (1998) and Carraro \& Munari (2004) acquired $B V I$ photometry for Pismis 19 stars. However, separate conclusions were reached regarding the cluster's fundamental parameters. Piatti et al. (1998) determined the following: $E(B-V)=$ $1.45 \pm 0.10, d=2.40 \pm 0.88 \mathrm{kpc}, \tau=1.0 \pm 0.2 \mathrm{Gyr}$, whereas Carraro \& Munari (2004) obtained $E(B-V)=1.48 \pm 0.15$, $d=1.5 \pm 0.4, \tau \simeq 0.8$ Gyr. The reddenings and distances agree within the mutual uncertainties, however, the individual uncertainties are large owing to the challenging task of analyzing highly obscured clusters solely via optical photometry. Carraro (2011) built upon Piatti et al. (1998) and Carraro \& Munari (2004) analyses by obtaining deeper photometry, and derived $d=2.5 \pm 0.5 \mathrm{kpc}$.

Individual reddenings for stars in Pismis 19 were determined as follows. Any point on the dereddening line (dl) for the $i$ th star is given by: $(J-H)_{i}=E_{J-H} / E_{H-K_{\mathrm{s}}} \times\left(H-K_{\mathrm{S}}\right)_{i}+b ; b=(J-H)_{i}-$ $E_{J-H} / E_{H-K_{\mathrm{s}}} \times\left(H-K_{\mathrm{s}}\right)_{i} ;(J-H)_{d l}=E_{J-H} / E_{H-K_{\mathrm{s}}} \times\left(H-K_{\mathrm{s}}\right)_{d l}+b$. The intersect between the dereddening line and the intrinsic relation was determined by minimizing the difference as a function of $\left(H-K_{\mathrm{s}}\right)_{0}:\left|(J-H)_{d l}-(J-H)_{0, z}\right|=E_{J-H} / E_{H-K_{\mathrm{s}}} \times\left(H-K_{\mathrm{s}}\right)_{0, z}+$ $(J-H)_{i}-E_{J-H} / E_{H-K_{\mathrm{s}}} \times\left(H-K_{\mathrm{s}}\right)_{i}-(J-H)_{0, z}$. The reddening vector $\left(E_{J-H} / E_{H-K_{\mathrm{s}}}\right)$ characterizing dust along the line of sight was derived by tracking deviations of red clump stars from their mean intrinsic color owing to extinction. The mean intrinsic color was adopted from Majaess et al. (2011b), who inferred the result from nearby red clump stars $(d \leq 50 \mathrm{pc})$ with revised Hipparcos parallaxes (van Leeuwen 2007). The reddening vector determined from red clump stars is $E(J-H) / E\left(H-K_{\mathrm{s}}\right)=2.02$. 


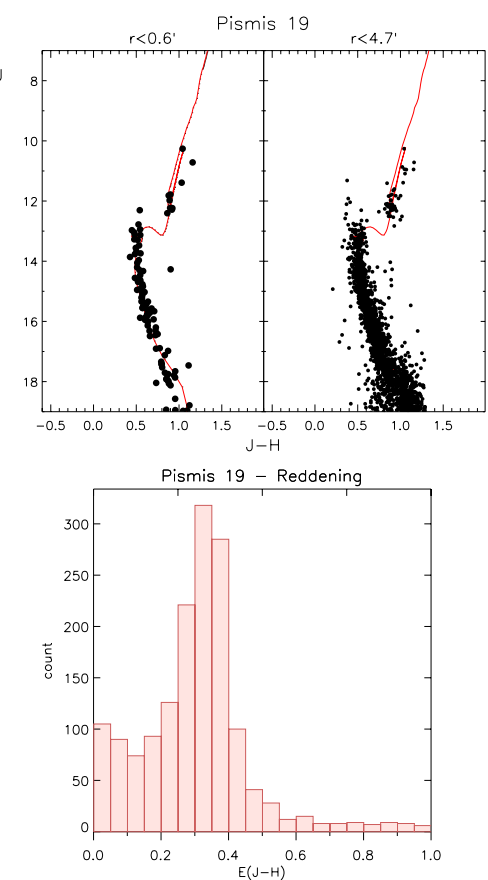

Fig. 2. VVV color-magnitude diagrams for Pismis 19 at varying radii. $J H K_{\mathrm{S}}$ Padova isochrones (Bonatto et al. 2004) were employed. Individual reddenings were determined via the approach outlined in Sect. 3.1. Photometric errors and binary contamination artificially broaden the breadth of the differential reddening.

That result agrees with a determination for the region from 2MASS photometry (Straižys \& Laugalys 2008). A reddening diagram was subsequently compiled (Fig. 2), and the mean reddening is $\langle E(J-H)\rangle=0.34 \pm 0.04$. The cluster stars terminate near F2 according to the intrinsic $J H K_{\mathrm{s}}$ relation of Straižys \& Lazauskaitè (2009).

A color-magnitude diagram was compiled for Pismis 19 stars surrounding J2000 coordinates of 14:30:40.54-60:53:32.2 (Fig. 2) ${ }^{1}$. A $\log \tau=9.05 \pm 0.10$ Padova isochrone (Bonatto et al. 2004) was adopted based on the reddening and spectral types inferred (Fig. 2), and since that age provides an evolutionary track which matches cluster members ranging from $\sim \mathrm{M} 0$ dwarfs to evolved stars. A precise fit was obtained owing to several factors. First, two of three free parameters associated with isochrone fitting were constrained by the color-color analysis, namely the reddening and age (spectral type at the turnoff). The remaining parameter (excluding metallicity) is the shift required in magnitude space to overlay the isochrone upon the data. The best fit and uncertainties were established via the traditional visual approach (e.g., Carraro \& Munari 2004; Bonatto \& Bica 2010), and the latter represents the limit where a mismatch is clearly perceived. Paunzen \& Netopil (2006, and references therein) note that errors tied to isochrone fitting via computer algorithms are comparable to those associated with the traditional approach. Secondly, field star contamination was mitigated since the surface density of cluster members is an order of magnitude larger, and furthermore, the cluster members occupy a heavily reddened locus separated from less-reddened field stars (Fig. 2). Lastly, the deep VVV photometry provided excellent anchor points for isochrone fitting.

The final parameters for Pismis 19 are: $d=2.40 \pm 0.15 \mathrm{kpc}$, $\langle E(J-H)\rangle=0.34 \pm 0.04$, and $\log \tau=9.05 \pm 0.10$. The distance is tied to a ratio of total to selective extinction $(R)$ derived by

\footnotetext{
1 The coordinates cited for the cluster center in simbad require updating.
}

Majaess et al. (2011a; see also Bonatto et al. 2004, and references therein). The distance derived here agrees with the latest estimate from optical photometry (Carraro 2011).

\subsection{NGC 4349}

Kholopov (1956) and Kraft (1957) noted that the 5.8 classical Cepheid R Cru may be a member of NGC 4349. That assessment was based in part on the Cepheid's proximity and brightness relative to cluster members. Cepheids are typically among the foremost evolved members of their host clusters. Lohmann (1961) employed $U B V$ photographic photometry to derive cluster parameters of: $d=1700 \mathrm{pc}$ and $\tau=600$ Myr. Fernie (1963) obtained photoelectric $B V$ photometry from the Cape Observatory and established a cluster distance of $d=900 \mathrm{pc}$, for $E(B-V)=$ 0.31 . The distance to NGC 4349 cited by Fernie (1963) is approximately half that derived by Lohmann (1961). Fernie (1963) concluded that R Cru is unassociated with NGC 4349 since the Cepheid lies towards the cluster's periphery. Cluster Cepheids known during that era had been discovered near the cluster center (e.g., S Nor/NGC 6087). Incidentally, the distance to NGC 4349 established by Fernie (1963) is consistent with that inferred for R Cru from present day period-Wesenheit relations (Majaess et al. 2011b). Lindoff (1968) revised the Lohmann (1961) age for NGC 4349 downward to $\log \tau=8.04$. Loktin \& Matkin (1994) computed the following properties for NGC 4349 based on a reanalysis of existing photometry: $d=2176 \mathrm{pc}$, $E(B-V)=0.384$, and $\log \tau=8.315$. In sum, published parameters for NGC 4349 span $d=900-2200$ pc.

A reddening vector of $E(J-H) / E\left(H-K_{\mathrm{s}}\right)=2.04$ was determined from red clump stars along the line of sight (see also Straižys \& Laugalys 2008). The reddening vector was subsequently adopted to establish a reddening of $E(J-H)=0.09 \pm$ 0.02. Stars catalogued by Lohmann (1961) as likely cluster members were employed to derive that result. New photoelectric $U B V$ photometry ${ }^{2}$ obtained for stars in NGC 4349 were likewise used to constrain the cluster reddening, and age. A comparison between that photoelectric $U B V$ photometry and the photographic photometry of Lohmann (1961) reveals the latter is offset from the standard system: $B-V=(1.02 \pm 0.02) \times(B-V)_{\mathrm{L} 61}-$ $0.02 \pm 0.02 ; U-B=(0.96 \pm 0.02) \times(U-B)_{\mathrm{L} 61}+0.09 \pm 0.01$; $V=(-0.015 \pm 0.03) \times(B-V)_{\mathrm{L} 61}+0.06 \pm 0.02+V_{\mathrm{L} 61}$. The offset may partly explain the difference between the distances inferred from the $U B V$ photometry of Lohmann (1961) and the present analysis. Applying an intrinsic $U B V$ color-color relation to the corrected data yields a reddening of $E(B-V)=0.32 \pm 0.03$. The canonical extinction law was employed, and may be refined once spectroscopic observations are available. Stars in NGC 4349 terminate near B8-A0 according to the intrinsic $J H K_{\mathrm{S}}$ and $U B V$ relations (e.g., Straižys \& Lazauskaitė 2009; Turner 2011). Published reddenings for R Cru (Fernie 1990) are nearly half that derived for the cluster, implying that the Cepheid lies in the foreground. That is consistent with the Cepheid's parameters as inferred from the latest period-Wesenheit relations (e.g., Benedict et al. 2007), which indicate that R Cru is less than $1 \mathrm{kpc}$ distant.

A color-magnitude diagram for NGC 4349 is shown in Fig. 3. A $\log \tau=8.55 \pm 0.10$ Padova isochrone (Bonatto et al. 2004) was adopted based on the reddening and spectral types inferred from the color-color diagram, and since that age provides an evolutionary track which aptly matches both bluer and redder evolved members. NGC 4349 features evolved stars

\footnotetext{
2 Obtained with the 0.6 m Helen Sawyer Hogg Telescope which was stationed at Cerro Las Campanas, Chile.
} 


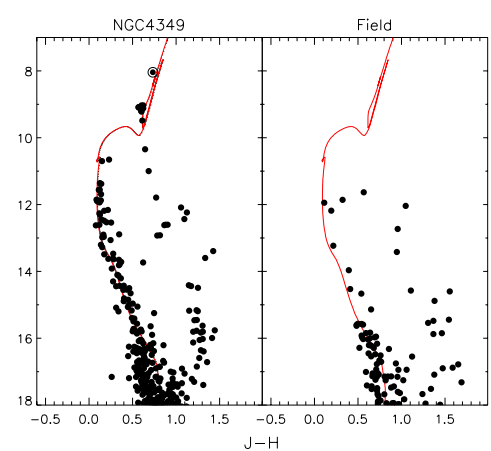

Fig. 3. Color-magnitude diagrams constructed for NGC 4349 and an adjacent comparison field using VVV/2MASS $J H K_{\mathrm{s}}$ photometry. The circled dot near the tip of the giant branch is TYC 8975-2606-1, which hosts a substellar companion (Lovis \& Mayor 2007). To mitigate contamination the CMDs feature stars within $r<1.2^{\prime}$. Seven evolved red stars beyond that radius were added to the CMD for NGC 4349.

brighter than the saturation limit of the VVV survey. Therefore, $J H K_{\mathrm{s}}$ photometry for these stars were taken from 2MASS. The color-magnitude diagram for NGC 4349 was restricted to stars within $\leq 1.2^{\prime}$ to mitigate field contamination. The final parameters for NGC 4349 are: $d=1.63 \pm 0.13 \mathrm{kpc}, E(J-H)=$ $0.09 \pm 0.02$, and $\log \tau=8.55 \pm 0.10$. A ratio of total to selective extinction $(R)$ was adopted from Majaess et al. (2011b) (see also Bonatto et al. 2004, and references therein). The distance and reddening agree with that established by Claria \& Lapasset $\left(1989, d=1.74 \pm 0.65 \mathrm{kpc}\right.$ and $\left.E_{B-V}=0.34 \pm 0.03\right)$.

In their comprehensive survey Lovis \& Mayor (2007) discovered that TYC 8975-2606-1 hosts a substellar companion (designated NGC 4349 127b). Lovis \& Mayor (2007) adopted cluster parameters of $d=2200 \mathrm{pc}$ and $\tau=200 \mathrm{Myr}$, which implied a $3.9 \mathcal{M}_{\odot}$ host. However, the distance and age established here for NGC 4349 are 30\% nearer and 150 Myr older, respectively. The parent star exhibits the following parameters according to the Padova isochrone applied: $\mathcal{M}_{*} / \mathcal{M}_{\odot} \sim 3.1$ and $\log L / L_{\odot} \sim 2.7$. Yet the principal source of uncertainty hindering a reliable determination of the orbital parameters remains the sparsely sampled radial velocity curve (Lovis \& Mayor 2007), as indicated by simulations conducted using the Systemic Console (Meschiari et al. 2009). Nevertheless, a minimum mass for the substellar companion of $\mathcal{M} / \mathcal{M}_{\mathcal{J}} \sim 17$ was obtained. Kashyap et al. (2008) derived an X-ray luminosity for the system in order to evaluate whether giant planets in close proximity to the host are catalysts for magnetic activity. That determination was based on a distance to NGC 4349 of $d=2176 \mathrm{pc}$, thereby reaffirming the importance of establishing a precise distance scale.

\section{Conclusion}

VVV $J H K_{\mathrm{s}}$ observations may be employed to help establish precise cluster distances that feature comparatively reduced uncertainties $(\leq 10 \%)$. That is illustrated by deriving fundamental parameters for Pismis 19 and NGC 4349, two important clusters which hitherto exhibit sizable uncertainties (60\%, Sects. 3.1 and 3.2). A precise distance determination for Pismis 19 from optical photometry was hampered in part by significant reddening (Fig. 2, $A_{V} \sim 4$ ). The existing ambiguity surrounding the distance to NGC 4349 ensured that the pertinence of invaluable putative constituents were mitigated (i.e., the classical Cepheid R Cru and a substellar companion for the member TYC 8975-2601-1). Parameters derived for Pismis 19 are: $d=2.40 \pm 0.15 \mathrm{kpc},\langle E(J-H)\rangle=0.34 \pm 0.04$, $\log \tau=9.05 \pm 0.10$ (Fig. 2), whereas NGC 4349 exhibits $d=1.63 \pm 0.10 \mathrm{kpc}, E(J-H)=0.09 \pm 0.02, \log \tau=8.55 \pm 0.10$
(Fig. 3). The nature of the VVV survey ensured that the revised results, which have pertinent ramifications, compliment existing estimates and display a marked improvement $(\geq 5 \times)$ in precision. New VVV $J H K_{\mathrm{s}}$ for stars in NGC 4349 and Pismis 19 imply that: existing physical parameters derived for NGC 4349 127b need to be redetermined in part since the mass for the host star was revised downward to $\mathcal{M}_{*} / \mathcal{M}_{\odot} \sim 3.1$ (Sect. 3.2); the classical Cepheid R Cru is not a member of NGC 4349 (Sect. 3.2); and VVV $J H K_{\mathrm{s}}$ photometry is particularly suited for constraining parameters of obscured and differentially reddened intermediateage clusters (e.g., Pismis 19, Fig. 2).

The VVV and UKIDSS surveys (Lucas et al. 2008; Minniti et al. 2010) may be employed to achieve significant gains toward strengthening the open cluster distance scale. However, considerable work remains, and improvements in the pipelines used to process the data are inevitable given the nascent nature of the aforementioned surveys.

Acknowledgements. D.M. is grateful to the following individuals and consortia whose efforts lie at the foundation of the research: 2MASS, P. Stetson (DAOPHOT), W. Lohmann, WEBDA (E. Paunzen), DAML (W. Dias), C. Lovis, M. Mayor, CDS, arXiv, and NASA ADS. We gratefully acknowledge use of data from the ESO Public Survey programme ID 179.B-2002 taken with the VISTA telescope, the Cambridge Astronomical Survey Unit, and funding from the FONDAP Center for Astrophysics 15010003, the BASAL CATA Center for Astrophysics and Associated Technologies PFB-06, the MILENIO Milky Way Millennium Nucleus from the Ministry of Economics ICM grant P07-021-F, and Proyecto FONDECYT Regular 1090213. W.G., C.M.B., and D.G. are grateful for support from the Chilean Center for Astrophysics FONDAP 15010003 and the BASAL Centro de Astrofisica y Tecnologias Afines (CATA) PFB-06/2007. R.K. acknowledges support from Proyecto DIUV23/2009, Universidad de Valparaíso. R.S. acknowledges financial support from CONICYT through GEMINI Project No. 32080016.

\section{References}

Benedict, G. F., McArthur, B. E., Feast, M. W., et al. 2007, AJ, 133, 1810 Bonatto, C., \& Bica, E. 2010, A\&A, 516, A81

Bonatto, C., Bica, E., \& Girardi, L. 2004, A\&A, 415, 571

Borissova, J., Bonatto, C., Kurtev, R., et al. 2011, A\&A, 532, A131

Carraro, G. 2011, A\&A, 536, A101

Carraro, G., \& Munari, U. 2004, MNRAS, 347, 625

Catelan, M., Minniti, D., Lucas, P. W., et al. 2011, RR Lyrae Stars, Metal-Poor Stars, and the Galaxy, 145

Claria, J. J., \& Lapasset, E. 1989, MNRAS, 241, 301

Cousins, A. W. J., \& Caldwell, J. A. R. 2001, MNRAS, 323, 380

Dias, W. S., Alessi, B. S., Moitinho, A., \& Lépine, J. R. D. 2002, A\&A, 389, 871 Fernie, J. D. 1963, The Observatory, 83, 33

Fernie, J. D. 1990, ApJS, 72, 153

Lindoff, U. 1968, Arkiv for Astronomi, 5, 1

Lohmann, W. 1961, Astron. Nachr., 286, 105

Loktin, A. V., \& Matkin, N. V. 1994, A\&AT, 4, 153

Lovis, C., \& Mayor, M. 2007, A\&A, 472, 657

Lucas, P. W., Hoare, M. G., Longmore, A., et al. 2008, MNRAS, 391, 136

Kashyap, V. L., Drake, J. J., \& Saar, S. H. 2008, ApJ, 687, 1339

Kholopov, P. N. 1956, Peremennye Zvezdy, 11, 325

Kraft, R. P. 1957, ApJ, 126, 225

Majaess, D., Turner, D., Lane, D., \& Krajci, T. 2011a, JAAVSO, 39, 219

Majaess, D., Turner, D., Moni Bidin, C., et al. 2011b, ApJ, 741, L27

Meschiari, S., Wolf, A. S., Rivera, E., et al. 2009, PASP, 121, 1016

Minniti, D., Lucas, P. W., Emerson, J. P., et al. 2010, New Astron., 15, 433

Minniti, D., Hempel, M., Toledo, I., et al. 2011, A\&A, 527, A81

Moni Bidin, C., Mauro, F., Geisler, D., et al. 2011, A\&A, 535, A33

Paunzen, E., \& Netopil, M. 2006, MNRAS, 371, 1641

Piatti, A. E., Clariá, J. J., Bica, E., Geisler, D., \& Minniti, D. 1998, AJ, 116, 801

Pietrzyński, G., \& Gieren, W. 2004, Variable Stars in the Local Group, IAU Colloq, 310, 87

Saito, R. K., Hempel, M., Minniti, D., et al. 2011, A\&A, in press

Straižys, V., \& Laugalys, V. 2008, Baltic Astron., 17, 253

Straižys, V., \& Lazauskaite, R. 2009, Baltic Astron., 18, 19

Turner, D. G. 1979, PASP, 91, 642

Turner, D. G. 2010, Ap\&SS, 326, 219

Turner, D. G. 2011, RMxAA, 47, 127

van Leeuwen, F. 2007, A\&A, 474, 653

van Leeuwen, F. 2009, A\&A, 497, 209 